Revue internationale P.M.E.

Économie et gestion de la petite et moyenne entreprise

Revure

internationale

PME

\title{
Préparation des PME familiales belges à la transmission et impact sur la performance
}

\section{Olivier Colot}

Volume 22, numéro 2, 2009

URI : https://id.erudit.org/iderudit/044032ar

DOI : https://doi.org/10.7202/044032ar

Aller au sommaire du numéro

Éditeur(s)

Presses de l'Université du Québec

ISSN

0776-5436 (imprimé)

1918-9699 (numérique)

Découvrir la revue

Citer cet article

Colot, O. (2009). Préparation des PME familiales belges à la transmission et impact sur la performance. Revue internationale P.M.E., 22(2), 95-132.

https://doi.org/10.7202/044032ar
Résumé de l'article

Une large littérature conceptuelle met en évidence les difficultés éprouvées lors de la transmission des entreprises familiales à la génération suivante. Ces développements théoriques ont été validés par de nombreuses études empiriques, même s'il subsiste des débats contradictoires. Les objectifs de cette recherche sont de déterminer si la préparation de la transmission a une influence sur la performance des PME familiales et si ces PME se heurtent à moins de problèmes. Une enquête, réalisée auprès de 2000 PME, a permis de relever 159 transmissions et de collecter les informations concernant la transmission. Cet article utilise la méthodologie du pairage statistique de manière à comparer les performances de PME familiales préparées à la transmission à celles d'homologues non préparées. Les résultats obtenus ne permettent pas de montrer que les PME familiales préparées sont significativement plus performantes. Les résultats montrent néanmoins une évolution des performances des PME non familiales après la transmission (observée sur trois ans) encore plus négative en cas de non-préparation.
Ce document est protégé par la loi sur le droit d'auteur. L'utilisation des services d'Érudit (y compris la reproduction) est assujettie à sa politique d'utilisation que vous pouvez consulter en ligne.

https://apropos.erudit.org/fr/usagers/politique-dutilisation/ 


\title{
Préparation des PME familiales belges à la transmission et impact sur la performance
}

Olivier COLOT

Université de Mons-Hainaut

MOTS CLÉS

Entreprise familiale - Préparation à la succession

Entrepreneuriat - PME - Performance

\begin{abstract}
L'AUTEUR
OLIVIER COLOT détient un doctorat en sciences de gestion et est chargé de cours à la Faculté warocqué de l'Université de Mons-Hainaut où il enseigne dans le domaine de la comptabilité et de l'audit. II assure la coordination de la Chaire entrepreneuriat. II est également membre du Centre de recherche warocqué et s'occupe de la coordination des documents d'économie et de gestion. Ses recherches concernent principalement les domaines de la comptabilité, de l'entreprise familiale et de l'entrepreneuriat.
\end{abstract}

\begin{abstract}
RÉSUMÉ
Une large littérature conceptuelle met en évidence les difficultés éprouvées lors de la transmission des entreprises familiales à la génération suivante. Ces développements théoriques ont été validés par de nombreuses études empiriques, même s'il subsiste des débats contradictoires. Les objectifs de cette recherche sont de déterminer si la préparation de la transmission a une influence sur la performance des PME familiales et si ces PME se heurtent à moins de problèmes. Une enquête, réalisée auprès de 2000 PME, a permis de relever 159 transmissions et de collecter les informations concernant la transmission. Cet article utilise la méthodologie du pairage statistique de manière à comparer les performances de PME familiales préparées à la transmission à celles d'homologues non préparées. Les résultats obtenus ne permettent pas de montrer que les PME familiales préparées sont significativement plus performantes. Les résultats montrent néanmoins une évolution des performances des PME non familiales après la transmission (observée sur trois ans) encore plus négative en cas de non-préparation.
\end{abstract}

\begin{abstract}
A large part of the literature shows the difficulty for family firms to operate their transmission to the following generation. These theoretical developments were validated by many empirical studies, even if they remain debatable. The aim of this research is to determine if the preparation of succession has an influence on the performance
\end{abstract}


of family SMEs, and to detect the principal succession problems of Belgian family SMEs. We therefore conducted a large inquiry in order to identify Belgian family SMEs and their main succession characteristics. This paper uses paired samples methodology to compare the performance of family SMEs prepared for succession with that of non-prepared family SMEs. The results do not show that the prepared family SMEs are significantly more competitive. The results show however an evolution in the performances of non-family SMEs after succession (over three years) that are even more negative if they are not prepared.

\section{RESUMEN}

Una amplia literatura conceptual subraya las dificultades encontradas en el momento de la transmisión de las empresas familiares a la generación siguiente. A pesar de que estos desarrollos teóricos fueran validados por numerosos estudios empíricos, aún permanecen en los debates. Los objetivos de esta investigación es determinar si la preparación de la sucesión tiene una influencia sobre el resultado de las PyMEs familiares y si estas PyMEs encuentran menos problemas. Una encuesta, realizada ante 2000 PyMEs, permitió identificar 159 transmisiones y recoger la información relativa a la transmisión. Este artículo utiliza la metodología de los pares estadísticos para comparar el resultado de PyMEs familiares preparadas a la transmisión con las de homólogas no preparadas. Los resultados obtenidos no permiten demostrar que las PyMEs familiares preparadas son significativamente más competitivas. Los resultados demuestran sin embargo una evolución, todavía más negativa en caso de no preparación, del resultado de las PyMEs no familiares después de la transmisión (observada durante tres años)

\section{ZUSAMMENFASSUNG}

Die Schwierigkeiten von Nachfolgeregelungen in Familienunternehmen sind in der Literatur vor allem durch konzeptionelle Beiträge eingehend publiziert worden. Die darin dargelegten theoretischen Konzepte sind auch durch zahlreiche empirische Studien validiert worden, wobei Widersprüche weiterhin im Raum bestehen bleiben. Die vorliegende Studie versucht die Fragen zu beantworten, ob die Vorbereitung zur Nachfolge Einfluss auf die Leistung des Familienunternehmens hat und inwiefern diese Unternehmen mit weniger Problemen konfrontiert werden. Durch eine Umfrage bei 2000 KMU konnten 159 Betriebe identifiziert werden, die im Nachfolgeprozess. Der statistische Vergleich der Leistungen von vorbereiteten und unvorbereiteten Familien-KMU erbrachte keinen signifikanten Unterschied. Jedoch fällt die negative Leistungsentwicklung von Nicht-Familien-KMU (beobachtet über drei Jahre) auf, die sich nicht auf den Übergangsprozess vorbereitet haben.

\section{Introduction}

La santé d'une région, d'un pays se reflète le plus souvent dans la prospérité et dans le dynamisme des petites et moyennes entreprises qui s'y développent, y exercent leur activité en fournissant des emplois directement ou en permet- 
tant la création d'emplois en amont et en aval de la chaîne économique. Or, chaque année, de nombreuses entreprises disparaissent ou déposent leur bilan en raison d'un problème de transmission. Ainsi, un grand nombre d'emplois sont perdus annuellement avec la fermeture d'entreprises qui n'ont pas su ou pu trouver une solution au problème de leur transmission.

Selon Langerock (1999) et Donckels (1989, p. 31), les principaux problèmes qui se posent lors de la succession sont le financement de la reprise, la fixation de la valeur, l'ensemble des aspects juridiques et fiscaux liés à la succession et les aspects humains, c'est-à-dire les émotions liées à la succession. D'autres recherches (Hirigoyen, 1988; Barach et Ganitsky, 1995) ont également mis en évidence les principales causes d'échecs en matière de transmission d'entreprises familiales: l'absence ou le manque de préparation, la personnalité du dirigeant, les conflits familiaux, la division du patrimoine familial, la complexité des montages juridiques, les droits de succession trop élevés, le manque d'expérience et de légitimité du successeur, une mauvaise préparation de la phase de transition, une vision stratégique différente et/ ou des barrières culturelles entre le chef d'entreprise et son successeur. Or, l'importance de la contribution des entreprises familiales n'est plus à prouver. Selon Kenyon-Rouviniez et Ward (2004), les entreprises familiales jouent un rôle considérable dans l'économie de la plupart des pays et représentent entre $50 \%$ et $90 \%$ du PIB de toutes les économies de marché. Elles représentent la forme d'entreprise la plus répandue du secteur privé, et ce, quel que soit le pays concerné (Cromie, Stephenson et Monteith, 1995; La Porta, Lopezde-Silanes et Shleifer, 1999; IFERA, 2003; Morck et Yeung, 2003; KenyonRouviniez et Ward, 2004 ; Diwisch, Voithofer et Weiss, 2006).

L'objectif de cette recherche est de mettre en évidence les principales difficultés et réticences rencontrées lors de la préparation de la transmission et de déterminer l'impact sur la performance. Sachant qu'un grand nombre d'entreprises ont été créées après la Seconde Guerre mondiale (Van Caillie, 1999, p. 3; Allouche et Amann, 2000, p. 48; Colli, 2003) et qu'elles sont confrontées ou vont bientôt l'être au problème de la transmission (OSÉO BDPME, 2005, p. 1), il est intéressant de chercher à mieux comprendre d'où viennent ces réticences de manière à mieux sensibiliser et à mieux préparer les futures transmissions d'entreprise. En effet, selon les estimations d'un groupe d'experts (Commission européenne, 2004, p. 7), un tiers des entreprises européennes feront l'objet d'une transmission dans les 10 prochaines années, soit entre $25 \%$ et $40 \%$ des entreprises selon les États membres. Ce chiffre correspond à une moyenne annuelle de 610000 transmissions de PME, dont 300000 employant du personnel et impliquant 2,1 millions d'emplois et 310000 n'employant pas de personnel. 
Vu l'importance de ces chiffres, il est surprenant de constater qu'aucune étude empirique approfondie n'a été réalisée en Belgique sur cette problématique. Cet article propose dès lors d'appliquer la méthodologie de l'appariement statistique à un échantillon de PME familiales belges préparées et non préparées à la transmission, de manière à comparer leurs rentabilités opérationnelle, économique et financière à celles d'homologues non familiales. Au préalable, cette recherche présente, dans un premier temps, l'importance des échecs de transmissions et, dans un second temps, les facteurs explicatifs des différences de préparation et des difficultés éprouvées lors de la transmission. Une enquête a également été réalisée en vue de recueillir des informations qualitatives concernant les transmissions, et les principaux résultats concernant la préparation et les difficultés sont présentés.

\section{1. Échec des transmissions d'entreprise}

Selon Birley (1986), environ $70 \%$ des entreprises familiales ne survivent pas jusqu'à la deuxième génération et $90 \%$,jusqu'à la troisième. Une étude longitudinale menée aux États-Unis par Ward (1997, p. 2) sur 200 entreprises familiales du secteur industriel et sur une période de 60 ans a montré que moins des deux tiers de celles-ci étaient encore entre les mains de la famille après la deuxième génération. Seulement $13 \%$ de ces entreprises ont conservé un caractère familial au-delà de la troisième génération. Il indique aussi que $5 \%$ d'entre elles sont vendues à des tiers et $2 \%$ sont entrées en Bourse et ne sont plus sous le contrôle de la famille. Pour expliquer cet important taux d'échec, l'auteur évoque trois raisons : la petite taille des entreprises familiales et leur manque de ressources financières, les conflits familiaux et l'absence de planification de la relève. Leach et Bogod (1999, p. 161) ont obtenu des résultats assez similaires pour l'Angleterre: seulement $24 \%$ des entreprises familiales survivent à la deuxième génération et seulement $14 \%$ atteignent la troisième génération. En France, une étude du BDPME (1998) portant sur 1472 transmissions d'entreprises entre 1993 et 1997 a montré que $22 \%$ d'entre elles échouent dans les cinq premières années et que près de $30 \%$ font de même dans les sept premières années. Pour la Belgique, aucune étude n'est disponible à ce sujet mais la situation n'est sans doute pas très différente. Le fait que très peu d'entreprises familiales survivent à la première génération est donc universel et indépendant du contexte culturel ou de l'environnement économique. Ce n'est d'ailleurs pas une coïncidence si l'espérance de vie moyenne d'une entreprise familiale est estimée à environ 24 ans (Beckard et Dyer, 1983). Aronoff (2001) estime que 30 \% des entreprises familiales réussissent le passage à la deuxième génération, que $10 \%$ à $15 \%$ passent à la troisième et que seulement $3 \%$ à $5 \%$ arrivent jusqu'à la quatrième génération. Selon Carlock et Ward (2001), un nombre significatif 
d'entreprises dans le monde est de type familial et peu d'entre elles survivent au changement de génération. De même, Brunello (2002) estime que $80 \%$ à $85 \%$ des entreprises familiales disparaissent avant la troisième génération. Plusieurs raisons sont évoquées pour expliquer ce taux d'échec si élevé. La principale cause est l'absence de planification de la transmission ou une planification inadaptée de celle-ci (Ward, 1988).

\section{Facteurs explicatifs}

\subsection{L'absence ou le manque de préparation}

Cette absence de planification a été soulignée par de nombreux auteurs (Christensen, 1953; Gasse, Théberge et Naud, 1988; Handler et Kram, 1988; Donckels, 1989; Noël, 1998). Déjà en 1953, Christensen, sur la base de son étude de 108 PME en croissance, avait mis en évidence un manque de préparation sérieuse et l'absence d'un calendrier. La plupart des entreprises familiales de la «première génération» ne survivent pas à leur fondateur par manque de préparation et parce qu'aucun plan n'a été établi (Donckels, 1989, p. 23). Dans une enquête menée par Donckels (1989), il est ressorti que deux tiers des chefs d'entreprise interrogés ne s'étaient pas encore préoccupés de leur succession, et ce, même si plus d'un tiers d'entre eux avaient déjà franchi le cap de la cinquantaine (1989, p. 31). Selon les résultats d'une enquête menée par Gauthier (1995) auprès de 300 propriétaires-dirigeants d'entreprises québécoises, 46,8 \% d'entre eux jugent opportun que leur successeur prenne la relève à leur retraite, $44,4 \%$ pensent que le successeur sera un membre de la famille mais 69,8\% n'ont pas prévu de plan de succession pour leur entreprise. En 1998, une enquête menée par Noël (1998, p. 40) sur 200 propriétaires-dirigeants d'entreprises canadiennes révèle que $81 \%$ n'ont établi aucun plan de succession, 7 \% sont en train de l'établir et $12 \%$ y pensent. De même, une étude ${ }^{1}$ récente menée au Canada montre que $66 \%$ des chefs d'entreprise n'ont pas établi de processus pour le choix du successeur et $70 \%$ n'ont pas encore choisi leur successeur alors que plus de la moitié de ces dirigeants envisagent de se retirer dans les 10 années à venir. En France, seuls $10 \%$ des entrepreneurs individuels et $33 \%$ des patrons de société de plus de 55 ans déclarent préparer leur transmission, tandis que $20 \%$ uniquement des cédants potentiels sollicitent des conseils (Barbot et Richomme-Huet, 2004).

1. Centre d'études et de recherches fiscales de Deloitte et Touche et University of Waterloo (1999), «Les entreprises familiales canadiennes sont-elles une espèce en voie de disparition? », Sondage sur l'aptitude des entreprises familiales canadiennes à assurer leur avenir, Samson Bélair Deloitte et Touche. 
Ce constat paraît surprenant pour les entreprises familiales puisque les dirigeants de ce type d'entreprises, qui sont souvent à la fois dirigeant et propriétaire, considèrent que la continuité au sein de la famille est une chose très importante (Chua, Sharma et Chrisman, 1999; Bégin, 2006). En outre, il apparaît que la plupart des entrepreneurs qui ont bâti une entreprise prospère souhaitent la transmettre à leur héritier (Kuratko, 1993, p. 132-136; Laidaoui, 1995, p. 22). Malheureusement, entre le désir et la réalité, il y a un grand écart. Nous pouvons dès lors nous étonner de ce manque de préparation à la transmission de l'entreprise familiale et, plus particulièrement, des petites entreprises (PME).

Handler et Kram (1988) ont développé un modèle théorique dans lequel sont répertoriées quatre sources de facteurs de résistance à la planification de la transmission. Ceux-ci sont d'ordre individuel, interpersonnel, organisationnel et environnemental, tous dépendants les uns des autres.

\section{FIGURE 1}

Modèle de résistance à la planification de la transmission

\begin{tabular}{|c|c|}
\hline Facteurs encourageant la résistance & Facteurs réduisant la résistance \\
\hline \multicolumn{2}{|c|}{ Individu } \\
\hline Bonne santé & Problèmes de santé \\
\hline Manque de centres d'intérêts & Autres centres d'intérêts \\
\hline Rétention du contrôle & Disposé à déléguer \\
\hline Peur de se retirer & Opportunité d'une nouvelle vie \\
\hline Pas d'avis externes & Demande de l'aide externe \\
\hline \multicolumn{2}{|c|}{ Interpersonnel } \\
\hline $\begin{array}{l}\text { Problème ou manque } \\
\text { de communication }\end{array}$ & $\begin{array}{l}\text { Bonne communication } \\
\text { Confiance élevée }\end{array}$ \\
\hline Confiance minimale & Héritiers impliqués dans l'entreprise \\
\hline Héritiers incompétents & Programmes de formation \\
\hline ou pas intéressés & Partage du pouvoir \\
\hline Formation minimale & Un enfant comme héritier potentiel \\
\hline Contrôle du pouvoir & \\
\hline $\begin{array}{l}\text { Plusieurs héritiers } \\
\text { venant d'un peu partout }\end{array}$ & \\
\hline \multicolumn{2}{|c|}{ Organisationnel } \\
\hline La culture bloque le développement. & La culture encourage la continuité. \\
\hline Stabilité dans la croissance & Crise organisationnelle imminente \\
\hline $\begin{array}{l}\text { Maintien des structures hiérarchiques } \\
\text { linéaires }\end{array}$ & Structures encourageant la délégation \\
\hline \multicolumn{2}{|c|}{ Environnemental } \\
\hline Environnement stable & Environnement turbulent \\
\hline Services très spécialisés & Services plus ou moins spécialisés \\
\hline
\end{tabular}

Source: Handler et Kram (1988, p. 375). 
Sharma, Christman et Chuoo (1998) ont également proposé un modèle des facteurs de résistance. Pour ce faire, ils ont réalisé une étude empirique auprès de 177 entreprises familiales canadiennes et ont relevé trois principaux facteurs : la capacité du prédécesseur à se retirer, la présence d'un successeur potentiel et la présence d'un conseil d'administration.

\subsubsection{Les freins psychologiques}

Le changement du dirigeant de l'entreprise constitue un cap difficile pour une entreprise. Souvent, le chef d'entreprise a des réticences psychologiques lors de la passation de pouvoir car cela signifie la fin de carrière mais aussi la fin d'une vie. En outre, il est parfois difficile au chef d'entreprise de se séparer du pouvoir et des privilèges qui accompagnent l'exercice de ce pouvoir.

En s'appuyant sur l'importance du rôle du propriétaire-dirigeant lors de la succession dans une entreprise familiale, Sonnefeld (1988) a établi cinq catégories de dirigeants en examinant leur attitude face à leur départ de la PME: les monarques, les généraux, les ambassadeurs, les gouverneurs et les hédonistes. Les monarques dirigeront la PME jusqu'au décès et ne la quitteront que s'ils y sont forcés. Les généraux ne la quittent aussi que s'ils y sont forcés mais, au moment du départ, ils feront tout pour que la transmission échoue, et ce, de manière à revenir à la tête de celle-ci pour la sauver. Les ambassadeurs, quant à eux, quittent volontairement l'entreprise et deviennent des conseillers pour les futurs dirigeants. Enfin, les gouverneurs quittent également volontairement l'entreprise mais gardent peu de contact avec l'entreprise après leur départ. Enfin, les hédonistes quittent l'entreprise pour s'intéresser à des activités, souvent culturelles, auxquelles ils n'avaient pas eu le temps de se consacrer.

Daigne et Joly (1987), quant à eux, constatent que plus le dirigeant est âgé, plus il lui est difficile d'envisager de se séparer de son entreprise, qui a représenté sa raison d'être et l'essentiel de son existence. Ils conseillent aux chefs d'entreprise de commencer l'opération de transmission dès l'âge de 50-55 ans car les éventuels héritiers sont eux aussi à un tournant de leur carrière professionnelle. En outre, ils disposeront d'un plus long délai qui permettra une meilleure transition. Ambrose (1983, p. 53) avait déjà obtenu des résultats identiques quelques années auparavant. Son enquête montre, d'une part, que les propriétaires âgés de plus de 70 ans justifient la non-préparation de la transmission de l'entreprise par le manque d'intérêt de leurs enfants à l'égard de l'entreprise et, d'autre part, que les propriétaires de moins de 50 ans trouvent leurs enfants trop jeunes pour leur succéder. Le constat est le même en Suisse où selon l'enquête menée par l'IMD-Lombard Odier en 
$2001,80 \%$ des entrepreneurs souhaitent que leur entreprise demeure dans la famille, mais $30 \%$ pensent que leurs enfants ne sont pas intéressés par la reprise (Bégin, 2006).

Les chefs d'entreprises ont donc tendance à rester le plus longtemps possible aux commandes de l'entreprise, avec pour conséquence que lorsqu'ils décident enfin de partir, leurs enfants ont souvent établi d'autres projets de carrière. La transmission de l'entreprise peut donc s'avérer être un échec si le processus de transfert n'est pas commencé assez tôt pour intéresser l'enfant apte à reprendre le flambeau. Dans cette même optique, Davis et Tagiuri (1989) ont montré que la relation professionnelle ne pose pas de problème lorsque le père est dans la cinquantaine et l'enfant, âgé entre 23 à 33 ans alors qu'elle peut être très problématique quand le père est dans la soixantaine et que l'enfant concerné a entre 34 et 40 ans. Cela s'explique par la complémentarité des intérêts de chacun. Lorsque le père est dans la cinquantaine, il prépare sa retraite de manière sereine et le fils, qui a dans la trentaine, est prêt à relever de nouveaux défis. Le père jouera alors le rôle de «mentor». En revanche, si le père est déjà dans la soixantaine, il y a de grandes chances qu'il ne quitte son poste que le jour de sa mort, ce qui peut créer des frustrations chez le successeur qui doit attendre.

L'ancien dirigeant peut donc montrer une certaine réticence à mener à bien le transfert de la propriété. Cela se traduit généralement par un manque de préparation de la transmission et l'absence d'un calendrier sérieux pour la gestion de la succession. La principale explication évoquée est la difficulté pour le chef d'entreprise à faire le «deuil» de son entreprise. Meier (2002) a mis en évidence cinq motifs de résistance culturelle et psychologique: la perte de pouvoir, la perte de légitimité professionnelle et sociale, le risque de déconstruction, la perte de repères et de sens, le refus de la vieillesse et de la mort.

\section{La perte de pouvoir}

L'abandon du pouvoir n'est jamais une chose facile, surtout lorsqu'il s'agit du fondateur de l'entreprise. Selon Vatteville (1994), de nombreux dirigeants éprouvent des difficultés à renoncer à leur statut de chef d'entreprise, synonyme de perte de pouvoir et d'influence sur l'entreprise. «La transmission constitue la fin d'une légitimité hiérarchique qui leur permettait d'exercer légalement des liens de subordination ou de domination sur leurs collaborateurs ou fournisseurs.»

Peay et Gibb Dyer (1989) ont réalisé une étude aux États-Unis sur 79 dirigeants-propriétaires. Ils ont relevé deux types de besoins de pouvoir: le besoin de pouvoir social et le besoin de pouvoir personnel. En cas de 
besoin de pouvoir social, le dirigeant laisse une certaine liberté ou initiative à ses collaborateurs. Le pouvoir et le bien de l'entreprise passent avant leur considération personnelle tandis que, dans le cas du besoin de pouvoir personnel, le dirigeant organise son entreprise en exerçant une domination et un contrôle sur ses collaborateurs. Dès lors, l'entreprise doit représenter son influence et son pouvoir. Cette étude a montré que les dirigeants du premier groupe ont plus de facilité à transmettre leur entreprise.

Selon Kittel (1996), le dirigeant ne parvient pas à lâcher prise pour deux raisons. D'une part, il ne peut renoncer à son statut de chef d'entreprise, qui correspond à une image de soi valorisante au regard d'autrui. D'autre part, il ne peut renoncer au plaisir d'exercer son pouvoir.

\section{La perte de légitimité professionnelle et sociale}

La transmission de l'entreprise entraîne, pour le chef d'entreprise, la perte de sa légitimité charismatique au sein de sa famille ainsi que la perte de sa légitimité sociale au sein de son environnement économique et professionnel. En effet, la direction de l'entreprise familiale confère au dirigeant une autorité naturelle auprès de ses travailleurs mais également au sein de sa famille. La succession a donc des répercussions aussi bien dans le système entreprise que dans le système famille.

\section{Le risque de «déconstruction»}

Pour le chef d'entreprise qui s'est investi dans celle-ci durant de nombreuses années, son entreprise constitue une extension de lui-même. Pour Kittel (1996, p. 176), «l'attachement est quasi viscéral avec son entreprise». Selon Pailot (1998), la résistance des chefs d'entreprise à la transmission provient d'un refus conscient ou inconscient de perdre un objet d'attachement central dans la construction de leur identité personnelle et sociale, à savoir l'entreprise. «Transmettre son entreprise revient alors pour le dirigeant à perdre un petit bout de soi.»

\section{La perte de repères et de sens}

Le dirigeant-propriétaire, qui s'est investi dans son entreprise durant de nombreuses années, a défini ses priorités et ses choix en fonction de celle-ci. Des habitudes se sont donc installées. À la suite de l'abandon de son entreprise, il peut se demander quelle est sa justification d'être et sa raison de vivre. Bref, quand le dirigeant quitte son entreprise, il perd un des éléments qui donnaient un sens à sa vie. 


\section{Le refus de la vieillesse et de la mort}

Souvent, le dirigeant peut se sentir éternel. La transmission de l'entreprise est souvent considérée par le dirigeant comme la fin d'une époque. «Renoncer à ce qui fut sa raison de vivre et se pénétrer de la certitude ultime (la mort)» (Vatteville, 1994, p. 19) est une décision très difficile à prendre et peut donc faire l'objet de réticences, même inconscientes.

\subsubsection{Le manque de compétences du dirigeant de l'entreprise}

La mise en place d'un processus de transmission fait intervenir de nombreuses dispositions légales et réglementaires. En effet, de nombreuses disciplines s'imbriquent entre elles: l'économie, le management stratégique, la finance (analyse des décisions de financement de la transmission), la gestion (gérer le changement), le droit (respect de la législation), la fiscalité (le choix d'une technique de transmission est influencé par la fiscalité), la gestion des ressources humaines (gérer les problèmes humains), etc. Confronté à des disciplines qu'il ne maîtrise pas convenablement, le chef d'entreprise se trouve souvent dans l'impossibilité de prendre une décision et renonce alors à élaborer un plan de succession.

\subsubsection{La famille}

Dans l'entreprise familiale, l'imbrication des systèmes famille et entreprise peut être la cause de nombreux problèmes car il est clair que les priorités de l'une ne sont pas celles de l'autre. La succession d'une telle entreprise est d'autant plus complexe et délicate puisqu'il y a des problèmes humains à résoudre. Il sera donc extrêmement important de tenir compte des objectifs et des attentes des membres de la famille, des employés et de toutes les autres personnes directement ou indirectement concernées.

Le passage à la troisième génération est un cap encore plus difficile à franchir car il y a souvent plus de membres de la famille qui souhaitent s'investir dans l'entreprise. Ces membres auront probablement des divergences d'opinions concernant la manière de travailler et l'avenir de l'entreprise familiale. De plus, lorsque le chef d'entreprise a plusieurs enfants qui sont candidats à la reprise de l'entreprise, il devra faire un choix. De même, si certains d'entre eux n'ont pas les compétences requises, il devra le leur annoncer. Alors, par crainte que ses enfants interprètent mal ce rejet, par exemple, comme un manque d'amour paternel, le chef d'entreprise risque fort d'être tenté de reporter sans cesse la discussion sur la transmission et la succession de l'entreprise. 


\subsubsection{Les travailleurs}

Certains anciens employés fidèles peuvent voir d'un mauvais œil le fait que le chef d'entreprise engage ses enfants dans l'entreprise familiale dans le but spécifique de les préparer à prendre la relève. En effet, pour eux, cela signifie la fin de leurs ambitions de carrière ou anéantit leurs possibilités d'avancement. Ils pourraient dès lors adopter des comportements nuisibles au bon déroulement du processus de transmission.

La transmission de l'entreprise représente un changement important qui risque de mettre en péril l'organisation mise en place à travers les années. Une résistance au changement risque donc d'apparaître car même s'il est nécessaire à la survie de l'entreprise, un changement est toujours difficilement accepté. Cette résistance provient généralement d'un manque d'information, d'incertitudes et de rigidité, l'entreprise fonctionnant selon ses habitudes, ses normes et les structures du passé. L'insécurité provient de la peur du changement et donc du futur. Pour minimiser cette résistance, il conviendra de fournir une information de qualité pour convaincre les intéressés de la nécessité du changement.

\subsubsection{Les motivations du prédécesseur ou de la famille}

Dans la plupart des cas, la motivation principale est la pérennité et la survie de l'entreprise, avant même d'assurer la pérennité du caractère familial. Néanmoins, il peut exister d'autres motivations, par exemple, la recherche d'une satisfaction propre au prédécesseur: donner ce que l'on a reçu (Mouline, 2000, p. 233-236). Selon Mouline, ces deux types de motivation influence la transmission. Ainsi, le désir de pérennité sera associé à une préparation minutieuse de la transmission tandis que dans le cas du désir de don, il y aura peu ou pas de préparation.

\subsection{Choix et préparation du successeur}

\subsubsection{Mauvais choix du successeur}

Le choix du successeur repose très souvent sur la subjectivité. Si le successeur n'a pas les compétences requises, la transmission de l'entreprise s'avérera être un échec. Le chef d'entreprise doit faire la sélection des candidats successeurs et prendre très au sérieux son analyse. S'il est vraiment préoccupé par la pérennité de son entreprise, il doit choisir la personne qui semble être la plus apte à reprendre le flambeau, c'est-à-dire qui a le profil, les aptitudes et des objectifs qui correspondent à ceux de l'entreprise. Il ne doit pas se 
laisser guider par la subjectivité. Donckels et Hoebeke (1991, p. 14) ont montré que $77 \%$ des dirigeants s'occupent personnellement du recrutement des travailleurs et en particulier du successeur, $40 \%$ le font seul et sans aide.

Selon Kotter (1982), les successeurs internes ont deux avantages eu égard aux repreneurs externes. D'une part, ils ont une meilleure connaissance de l'entreprise: ses produits, ses marchés, ses concurrents et clients, son personnel, sa technologie et son organisation (Parrino, 1997). D'autre part, ils possèdent déjà des réseaux sociaux à partir desquels ils acquièrent l'information spécifique.

\subsubsection{Mauvaise préparation du successeur}

Ce cas de figure se présente souvent lorsque le successeur reprend le flambeau sans avoir acquis une expérience managériale au préalable. Même si le successeur travaillait dans l'entreprise familiale, il n'a pas souvent eu la possibilité de prendre des décisions et d'assumer des tâches et des responsabilités managériales car le père chef d'entreprise refusait toute délégation de pouvoir. Entre deux générations, il y a un fossé de 25 ans. Celui-ci implique une différence d'expérience, de maturité, de formation et d'échelle de valeurs (Donckels, 1989, p. 77).

\subsubsection{Les divergences d'intérêts des parties concernées}

Même si le processus de transmission a été bien planifié et que le successeur est compétent, des problèmes peuvent survenir lors de la mise en œuvre de la transmission de l'entreprise et même au-delà de celle-ci à cause de divergences d'intérêts des parties en présence (la famille, les actionnaires de la famille actifs et non actifs, les travailleurs, les clients, les banquiers, les fournisseurs, etc.). En effet, il n'est pas rare que la famille intervienne dans l'entreprise afin que la gestion soit orientée en fonction des intérêts de la famille. De même, les actionnaires familiaux actifs préfèrent un réinvestissement des bénéfices et une faible distribution de dividendes. Il y aura donc un conflit d'intérêts avec les actionnaires familiaux non actifs qui souhaitent recevoir un dividende élevé. En ce qui concerne les banquiers et les clients, fournisseurs, la relation de confiance privilégiée avec le chef d'entreprise est rompue et ils se posent des questions sur le successeur. L'environnement externe aura donc une influence sur les deux sous-systèmes que sont la famille et l'entreprise.

Un autre problème pouvant être mis en évidence par la théorie de l'agence peut également survenir lors du processus de transmission. Pendant la phase de transition, le prédécesseur est toujours propriétaire de l'entreprise 
familiale alors que le successeur devient le manager (un agent) de l'entreprise. Cette fonction d'agent peut susciter des conflits entre le prédécesseur et le successeur si ce dernier a des comportements sous-optimaux pour les actionnaires.

\subsection{Le choix d'une technique de transmission trop coûteuse}

Lors du choix de la technique de transmission, le chef d'entreprise doit tenir compte du coût financier que la société ou le successeur devra supporter. En effet, ils seront appelés à payer de lourds montants, aussi bien pour acquitter les droits de succession que pour verser une éventuelle indemnisation aux autres héritiers qui n'auraient pas reçu assez. Une situation idéale pour le père chef d'entreprise serait de disposer d'un gros patrimoine familial qui pourrait être réparti entre tous ses héritiers en donnant la majorité de l'entreprise familiale au successeur choisi. Si ce n'est pas le cas et si le successeur ne dispose pas de liquidités suffisantes pour financer la reprise, il devra recourir à l'endettement et se tourner vers les banquiers. Si cet endettement est trop lourd à supporter, il pourra se faire avancer l'argent par la société; mais, dans ce cas, l'entreprise risque d'être affaiblie par manque de liquidités.

Cette revue de la littérature sur la problématique de la transmission des PME dont le propriétaire cumule la fonction de dirigeant nous a permis de mettre en évidence les principales variables explicatives liées, soit à la personnalité du dirigeant, soit à d'autres éléments tels que le fonctionnement de la PME ou encore son environnement immédiat. L'objectif de cette recherche est double:1) relever les principales difficultés et réticences rencontrées lors la préparation de la transmission et vérifier si elles sont conformes à celles mentionnées dans la littérature;2) déterminer leur impact sur la performance des PME après l'opération de transmission.

\section{Méthodologie}

Les données financières ont été extraites du cédérom Belfirst ${ }^{2}$. Quant aux informations permettant d'identifier les entreprises familiales, l'absence d'une base de données satisfaisante a justifié le recours à la technique de l'enquête par questionnaire ${ }^{3}$ de manière à collecter les informations requises ${ }^{4}$.

2. Comptes annuels des entreprises belges soumises à l'obligation de dépôt auprès de la Banque nationale de Belgique.

3. Le questionnaire de l'enquête peut être consulté en annexe.

4. Dans un souci de concordance et de cohérence maximales avec le contexte de référence des entreprises belges, la forme, l'ordonnancement, le contenu ainsi que 


\subsection{Hypothèse}

De nombreuses études concluent que l'une des principales causes de l'échec d'une transmission est l'absence de planification (Ward, 1988; Hirigoyen, 1988; Donckels, 1989; Vatteville, 1994; Barack et Ganitsky, 1995; Meier, 2002). Dès lors, nous pouvons raisonnablement en déduire que la préparation ou non de l'opération de transmission aura une influence sur les performances des PME. Sur la base de ces arguments, nous pouvons émettre l'hypothèse suivante:

Hypothèse. La performance commerciale, économique et financière d'une PME familiale après la transmission varie de manière négative lorsque l'opération de transmission n'a pas été préparée.

\subsection{Population visée}

De manière à atteindre un maximum d'entreprises familiales, la recherche a été axée sur les PME, qui sont très souvent de nature familiale. En effet, la PME familiale est un type de sociétés dominant sur le marché belge (Donckels et Aerts, 1993, p. 162-164; Wtterwulghe et al. 1994; Van Gils, Voordeckers et Van Den Heuvel, 2004, p. 588), comme partout dans le monde (Allouche et Amann, 2000) et pourtant peu étudié (Van Gils, Voordeckers et Van Den Heuvel, 2004, p. 588). L'enquête a ainsi été réalisée auprès de l'ensemble des PME belges, créées avant le 31 décembre 1990, et ce, pour deux raisons: d'une part, de manière à ce que le caractère familial ou non soit bien imprégné dans l'entreprise et, d'autre part, de manière à toucher un maximum de PME ayant déjà fait l'objet d'une transmission. À l'intérieur de ce groupe de PME belges, il a été procédé à un échantillonnage aléatoire simple sans remise de 2000 entreprises. Une relance de l'enquête a été réalisée trois mois après le premier envoi.

\subsection{Descriptif de l'échantillon}

L'enquête a finalement donné lieu à 391 réponses exploitables, soit un taux de réponse de 23,86\%. Par ailleurs, la représentativité statistique de cet échantillon a été testée à partir de trois critères: la localisation géographique, le secteur d'activité et l'effectif (nombre de travailleurs). Au seuil de probabilité $\alpha$ de $5 \%$, l'hypothèse nulle de distributions identiques ne peut être rejetée pour chacune des variables prises en considération.

la terminologie du questionnaire ont été déterminés à l'issue d'un prétest auprès d'acteurs du terrain (issus de secteurs d'activité différents). 
Pour déterminer si les PME de l'échantillon sont familiales ou non, il a été considéré que l'entreprise est familiale lorsqu'elle satisfait à au moins deux des trois critères suivants:

- une famille détient au moins $50 \%$ des actions de l'entreprise;

- une famille a une influence décisive sur la stratégie de l'entreprise et sur les décisions de transmission de l'entreprise;

- la majorité du conseil d'administration est composée de membres d'une famille.

Nous avons choisi cette définition de la PME familiale essentiellement pour deux raisons. D'une part, cette définition de la PME familiale présente l'avantage de reposer sur des critères clairs et mesurables, par opposition aux définitions qualitatives qui sont plus subjectives et arbitraires. D'autre part, la définition est très proche des plus récentes définitions de l'entreprise familiale.

Ainsi, parmi les 391 entreprises de notre échantillon, 318 peuvent être considérées comme familiales. Avec un taux de 81,33\% de PME familiales (statistiquement significatif à un seuil de $1 \%$ ), nous obtenons des résultats similaires aux études précédentes menées sur l'importance des PME familiales en Belgique (Wtterwulghe et al., 1994, soit 82 \% ; Jorissen et al., 2002, soit $82,8 \%$ ). Par ailleurs, il convient de noter que l'enquête nous a permis de relever 159 transmissions de PME, dont 130 de nature familiale.

\subsection{Pairage statistique}

Pour analyser la performance des PME familiales et non familiales après l'opération de transmission et selon qu'elles y étaient préparées ou non, nous allons les comparer à des entreprises aussi similaires que possible, excepté qu'elles n'ont pas fait l'objet d'une transmission. Nous travaillerons donc sur des échantillons appariés. Cette technique permet entre autres d'éliminer les données démographiques (taille, secteur d'activité). En effet, selon Jorissen et al. (2002), les études comparatives sur les entreprises familiales et non familiales ignorent souvent les données démographiques (localisation, taille, âge, secteur) qui peuvent biaiser les résultats bivariés mettant en évidence les différences concernant le management et la performance entre ces deux types d'entreprises. Pour Westhead et Cowling (1998), les études qui ne contrôlent pas ces variables n'enregistrent pas de réelles différences de performances et de management entre les entreprises familiales et non familiales mais bien des différences dues aux données démographiques de l'échantillon. 
Pour procéder au pairage, il convient de choisir des «critères jugés pertinents, de manière à s'assurer que l'effet mesuré provient de la (ou des) variables(s) étudiée(s) et non de la différence de composition d'échantillons» (Thiétart,1999, p. 198). Si l'on se réfère à d'autres études empiriques utilisant cette méthode (Caby,1994), on constate que l'activité économique et la taille (estimée par l'effectif et/ou l'actif total) font l'unanimité. On sait en effet que certains indicateurs comptables sont sensibles à l'appartenance sectorielle et à la taille de l'entreprise considérée (Ooghe et Van Wymeersch, 1990, p. 395). En ce qui concerne le choix du critère de taille, le total bilantaire a été privilégié (il s'agit d'une des trois références à la taille des entreprises selon la loi comptable belge, avec le chiffre d'affaires et l'effectif). À ces deux critères (secteur d'activité et total du bilan), nous en avons ajouté un troisième qui permet de distinguer la PME familiale de la non familiale. L'échantillon de contrôle sera donc déterminé comme suit:

- PME familiale ou non;

- le secteur d'activité: code NACEBEL à quatre chiffres ${ }^{5}$;

- la taille: le total de l'actif ne peut pas varier de plus ou moins $20 \%{ }^{6}$.

Pour le pairage des entreprises, nous avons donc tenu compte du caractère familial de l'entreprise. Ainsi, nous avons pairé les PME familiales ayant fait l'objet d'une transmission avec des PME familiales n'ayant pas encore fait l'objet d'une transmission et les PME non familiales ayant fait l'objet d'une transmission avec des PME non familiales n'ayant pas encore fait l'objet d'une transmission. Pour chaque PME transmise, nous avons recherché l'ensemble des PME non transmises du même profil qui pouvaient convenir parmi les PME constituant notre échantillon de PME non transmises. En effet, comme les comptes annuels ne donnent aucune information concernant la transmission, nous avons donc identifié les PME non transmises sur la base des réponses à notre enquête.

Nous avons ainsi obtenu 102 paires «une PME transmise / une PME non transmise», dont 78 à caractère familial et 24 à caractère non familial.

5. Il a parfois été nécessaire d'assouplir ce critère de sélection. Le code NACEBEL à trois chiffres a été utilisé pour 23 entreprises et un autre à deux chiffres pour 34.

6. Lorsque plusieurs entreprises non transmises correspondaient au profil recherché, celle présentant le total bilantaire le plus proche de celui de sa jumelle transmise a été retenue. 


\subsection{Test de comparaison}

L'échantillon des PME transmises a été comparé à l'échantillon témoin (PME non transmises) au moyen d'un test statistique qui permet de comparer des observations appariées. Ainsi, pour chaque variable étudiée, il faut calculer les différences entre les paires en soustrayant systématiquement de la valeur du ratio de la PME transmise la valeur correspondante pour la PME témoin. Le test de comparaison est en fait pratiqué sur la moyenne des différences entre valeurs appariées. Un test de Student permet d'accepter ou de rejeter l'hypothèse nulle, comme le recommandent Cooper et Schindler (2006, p. 505).

Cette méthode ne suppose pas la normalité des distributions (AFNOR, 1988, p. 366; Heldenbergh, 1999, p. 205), ce qui est particulièrement intéressant dans la mesure où de nombreux ratios ne sont pas distribués normalement (Ooghe et Van Wymeersch, 1990, p. 392). Par ailleurs, il est conseillé d'éliminer les différences aberrantes. En effet, une trop grande différence pourrait être due à un élément isolé, indépendant des caractéristiques des sociétés transmises. Le test de Cochran permet précisément d'éliminer ces valeurs extrêmes. Pour chaque indicateur retenu, nous avons calculé les différences des paires d'entreprises (appelées Di). Ensuite, à partir des valeurs Di, nous avons calculé des valeurs Gi correspondant au rapport des $\mathrm{Di}^{2}$ de chaque paire sur la somme des $\mathrm{Di}^{2}$ de l'ensemble des paires pour un indicateur. Nous avons éliminé les valeurs Di auxquelles correspondait un Gi >0,12.

\subsection{Choix des indicateurs de performance}

En fonction du caractère non boursier de l'échantillon (la Belgique ne comporte que très peu de PME cotées), la dimension comptable de la performance financière est privilégiée, plus particulièrement à travers des paramètres de rentabilité. Il s'agit d'indicateurs de rentabilité relativement classiques qui permettent d'effectuer une analyse multidimensionnelle de la performance comptable des entreprises considérées : performance opérationnelle et commerciale (marges sur vente, valeur ajoutée), performance économique de l'activité (ROA) et performance financière (ROE). Pour permettre l'analyse des données, nous avons classé les entreprises dans un intervalle de temps compris entre $n-2$ et $n+3$, où $n$ correspond à l'année de la transmission. Selon Leker et Salomo (2000), la transmission aurait un impact sur la performance jusqu'à un maximum de deux ans après. Après trois années, une dégradation de la performance ne serait plus due directement à l'opération de transmission (choix de la technique, degré de préparation, choix du successeur, etc.) mais, par exemple, à une mauvaise gestion du successeur. 


\section{Résultats}

\subsection{Observation sur l'échantillon}

TABLEAU 1

Principales difficultés éprouvées lors de la transmission de l'entreprise

\begin{tabular}{|c|c|c|c|c|}
\hline Technique & & Fréquence & Pourcentage & $\begin{array}{c}\text { Pourcentage } \\
\text { valide }\end{array}$ \\
\hline \multirow{2}{*}{ Aucune difficulté. } & oui & 64 & $40,3 \%$ & $48,9 \%$ \\
\hline & non & 67 & $42,1 \%$ & $51,1 \%$ \\
\hline \multirow{2}{*}{ Difficulté de trésorerie. } & oui & 30 & $18,9 \%$ & $22,9 \%$ \\
\hline & non & 101 & $63,5 \%$ & $77,1 \%$ \\
\hline \multirow{2}{*}{ Adhésion du personnel. } & oui & 18 & $11,3 \%$ & $13,7 \%$ \\
\hline & non & 113 & $71,1 \%$ & $86,3 \%$ \\
\hline \multirow{2}{*}{$\begin{array}{l}\text { Appareil productif obsolète } \\
\text { ou non compétitif. }\end{array}$} & oui & 15 & $9,4 \%$ & $11,5 \%$ \\
\hline & non & 116 & $73,0 \%$ & $88,5 \%$ \\
\hline \multirow{2}{*}{ Endettement trop lourd. } & oui & 12 & $7,5 \%$ & $9,2 \%$ \\
\hline & non & 119 & $74,8 \%$ & $90,8 \%$ \\
\hline \multirow{2}{*}{$\begin{array}{l}\text { Entreprise trop influencée par la } \\
\text { personnalité du chef d'entreprise. }\end{array}$} & oui & 12 & $7,5 \%$ & $9,2 \%$ \\
\hline & non & 119 & $74,8 \%$ & $90,8 \%$ \\
\hline \multirow{2}{*}{$\begin{array}{l}\text { Pas de volonté du chef } \\
\text { d'entreprise de passer la main. }\end{array}$} & oui & 12 & $7,5 \%$ & $9,2 \%$ \\
\hline & non & 119 & $74,8 \%$ & $90,8 \%$ \\
\hline \multirow{2}{*}{$\begin{array}{l}\text { Manque d'accompagnement } \\
\text { du chef d'entreprise envers } \\
\text { son successeur. }\end{array}$} & oui & 12 & $7,5 \%$ & $9,2 \%$ \\
\hline & non & 119 & $74,8 \%$ & $90,8 \%$ \\
\hline \multirow{2}{*}{$\begin{array}{l}\text { Pas de préparation } \\
\text { de la transmission. }\end{array}$} & oui & 10 & $6,3 \%$ & $7,6 \%$ \\
\hline & non & 121 & $76,1 \%$ & $92,4 \%$ \\
\hline \multirow{2}{*}{$\begin{array}{l}\text { Manque d'information et de } \\
\text { communication dans la famille. }\end{array}$} & oui & 10 & $6,3 \%$ & $7,6 \%$ \\
\hline & non & 121 & $76,1 \%$ & $92,4 \%$ \\
\hline \multirow{2}{*}{ Autres difficultés. } & oui & 9 & $5,7 \%$ & $6,9 \%$ \\
\hline & non & 122 & $76,7 \%$ & $93,1 \%$ \\
\hline \multirow{2}{*}{$\begin{array}{l}\text { Difficultés commerciales } \\
\text { à la suite de la perte de clients } \\
\text { ou d'un fournisseur. }\end{array}$} & oui & 5 & $3,1 \%$ & $3,8 \%$ \\
\hline & non & 126 & $79,1 \%$ & $96,2 \%$ \\
\hline \multirow{2}{*}{$\begin{array}{l}\text { En cas de revente: } \\
\text { valorisation trop élevée. }\end{array}$} & oui & 5 & $3,1 \%$ & $3,8 \%$ \\
\hline & non & 126 & $79,1 \%$ & $96,2 \%$ \\
\hline \multirow{2}{*}{$\begin{array}{l}\text { Manque de recours } \\
\text { à des personnes spécialisées. }\end{array}$} & oui & 4 & $2,5 \%$ & $3,1 \%$ \\
\hline & non & 127 & $79,9 \%$ & $96,9 \%$ \\
\hline \multirow{2}{*}{$\begin{array}{l}\text { Le successeur avait une mauvaise } \\
\text { connaissance du secteur. }\end{array}$} & oui & 3 & $1,9 \%$ & $2,3 \%$ \\
\hline & non & 128 & $80,5 \%$ & $97,7 \%$ \\
\hline Total & & 131 & $82,4 \%$ & $100,0 \%$ \\
\hline Absents & & 28 & $17,6 \%$ & \\
\hline Total & & 159 & $100,0 \%$ & \\
\hline
\end{tabular}


De notre enquête, il ressort que près de $50 \%$ des PME ne se sont heurtées à aucune difficulté lors de leur transmission. Les principaux problèmes relevés par les autres PME interrogées étaient les difficultés de trésorerie avec $22,9 \%$, l'adhésion du personnel avec 13,7\%, un appareil productif obsolète ou non compétitif avec $11,5 \%$, un endettement trop lourd, un entreprise trop influencée par la personnalité du chef d'entreprise, pas de volonté du chef d'entreprise de passer la main ou encore un manque d'accompagnement du chef d'entreprise envers son successeur pour 9,2\%.

Les principales difficultés mises au jour dans cette enquête correspondent bien à celles de la littérature. En effet, les problèmes de trésorerie sont fréquents et trouvent leurs origines à divers niveaux: choix d'une technique de transmission trop coûteuse, choix d'un successeur incompétent, etc. L'adhésion du personnel est également un problème qui se pose fréquemment lors de la transmission. Certains travailleurs de l'entreprise voient parfois d'un mauvais œil l'arrivée du successeur, et ce, pour diverses raisons, par exemple si le successeur n'a pas fait ses preuves envers le personnel ou si les enfants n'ont été engagés dans l'entreprise familiale que dans le but de les préparer à prendre la relève. Comme pour certains, cela signifie la fin de leurs ambitions de carrière ou de leurs possibilités d'avancement, ils pourraient être enclins à adopter des comportements nuisibles au bon déroulement du processus de transmission.

TABLEAU 2

Problèmes de transmission et préparation de la PME familiale*

\begin{tabular}{|c|c|c|c|c|}
\hline \multirow{2}{*}{ Préparée? } & \multicolumn{3}{|c|}{ Aucune difficulté } & \multirow{2}{*}{ Total } \\
\hline & vrai & faux & pourcentage & \\
\hline oui & 19 & 22 & $46,34 \%$ & 41 \\
\hline non & 35 & 32 & $52,24 \%$ & 67 \\
\hline Total & 54 & 54 & $50,00 \%$ & 108 \\
\hline
\end{tabular}

* Statistiquement non significatifs.

Les PME familiales éprouvent-elles moins de problèmes lorsqu'elles ont été préparées à la transmission? Nous ne pouvons nous prononcer à la vue de ce tableau. En effet, seulement 46,34\% des PME familiales préparées n'ont pas eu de problèmes lors de la transmission pour 52,24\% de celles qui n'étaient pas préparées. Ce résultat est surprenant (mais de manière non significative et qu'il convient de restreindre à l'échantillon). Nous nous attendions plutôt à découvrir que la majorité des PME familiales n'ayant pas eu de difficultés lors de la transmission soient celles qui s'y étaient préparées. Nous verrons si ces résultats sont confirmés par les tests de performance. 
Nous avons ensuite observé si les PME familiales éprouvaient moins ou plus de problèmes lors de la transmission que les PME non familiales.

\section{TABLEAU 3}

Les PME familiales ont-elles moins ou plus de problèmes lors de la transmission que les PME non familiales?*

\begin{tabular}{lcccr}
\hline \multirow{2}{*}{ PME } & \multicolumn{3}{c}{ Aucune difficulté } & \multirow{2}{*}{ Total } \\
\hline familiale & 54 & faux & pourcentage & \\
non familiale & 10 & 54 & $50,00 \%$ & $\mathbf{1 0 8}$ \\
Total & $\mathbf{6 4}$ & $\mathbf{6 7}$ & $43,48 \%$ & $\mathbf{2 3}$ \\
\hline
\end{tabular}

* Statistiquement non significatif.

Ce tableau nous montre que les PME familiales ont un peu moins de difficultés à résoudre lors de leur transmission que les PME non familiales (résultats limités à l'échantillon). Cela peut entre autres s'expliquer par le fait que dans les PME familiales, les repreneurs font souvent partie de la famille et il est fort probable qu'ils travaillent dans l'entreprise. Ils ont dès lors une meilleure connaissance de l'entreprise et en ont sans doute déjà acquis les connaissances spécifiques.

Enfin, dans ce dernier tableau, nous avons observé qui était le repreneur, de manière à voir si, à la suite de l'opération de transmission, la PME a conservé ou non le caractère familial.

\section{TABLEAU 4}

Qui est le repreneur de la PME familiale ?*

\begin{tabular}{lcc}
\hline & \multicolumn{2}{c}{ PME familiales } \\
& Fréquence & Pourcentage \\
\hline Transmission aux enfants & 79 & $72,48 \%$ \\
Transmission à la famille élargie & 9 & $8,26 \%$ \\
Transmission aux salariés & 1 & $0,92 \%$ \\
Transmission à une personne & 16 & $14,68 \%$ \\
extérieure à l'entreprise & & $3,67 \%$ \\
Autre repreneur & 4 & $\mathbf{1 0 0 , 0 0} \%$ \\
Total & $\mathbf{1 0 9}$ & \\
\hline
\end{tabular}

* Statistiquement significatif à un seuil de $1 \%$.

Pour les PME familiales, la transmission s'effectue au sein de la famille dans un peu plus de $80 \%$ des cas. Nous pouvons en tirer comme conclusion qu'une PME familiale sur cinq perd son caractère familial lors de la phase de transmission. Pourquoi? Soit parce que le dirigeant n'a pas d'enfant $(5,2 \%$ 
des dirigeants), soit que le ou les enfants ne sont pas capables de reprendre la PME familiale ou encore qu'ils n'y sont pas intéressés. Dans ces différents cas, la PME sera transmise aux salariés $(1 \%)$ et pour la majorité, à une personne externe (19\%). Dans leur enquête, Laveren et Bisschops (2004) ont demandé aux dirigeants d'entreprises familiales flamandes à qui ils souhaitaient transmettre leur entreprise. Les réponses des 51 dirigeants qui étaient déjà décidés sur la question sont similaires à celles que nous avons obtenues: $64,71 \%$ des entreprises seront transmises aux enfants, 9,8\% à la famille élargie, 1,96\% aux salariés et $23,53 \%$ à un repreneur externe.

\subsection{Résultat des tests ${ }^{7}$}

La comparaison des performances entre les PME familiales préparées à la transmission et les PME familiales non préparées ne fait pas apparaître de réelles différences. En effet, nous constatons une amélioration des performances en général pour les deux groupes d'entreprises. Nous devons rejeter notre hypothèse, et ce, malgré les nombreux résultats statistiquement significatifs obtenus. Toutefois, pour chaque résultat statistiquement significatif, les valeurs des PME familiales transmises sont supérieures à celles des PME familiales non transmises pour les années qui suivent la transmission, excepté la marge brute d'exploitation entre $n-1$ et $n+1$ pour les PME non préparées et la rentabilité financière brute entre $n-1$ et $n+1$ pour les PME préparées.

Une des rares différences constatées se situe au niveau de l'évolution de la marge brute d'exploitation: elle est négative pour les PME familiales transmises non préparées et positive pour les préparées (résultat statistiquement significatif).

Ces résultats confirment ceux obtenus lors de notre enquête et illustrés au tableau 2. Cependant, il faut reconnaître que notre échantillon n'est constitué que de PME encore en activité. Dès lors, toutes les PME qui n'étaient pas préparées à la transmission et qui ont cessé leurs activités n'apparaissent pas dans nos résultats. La BDPME (1998, p. 7) souligne néanmoins que les transmissions mal ou pas du tout préparées sont 1,5 fois plus risquées que celles ayant fait l'objet d'une préparation. Mais même si la non-préparation de cette opération pourrait constituer une explication, l'impact dans les chiffres n'a pu être mis en évidence.

7. Le tableau 4 comprend les indicateurs de performance, tandis que les tableaux 5 et 6 montrent les évolutions entre $n-1$ et $n+1$ (CT), entre $n-2$ et $n+3$ (LT), et la moyenne avant et après transmission de l'ensemble des indicateurs, $n$ étant l'année de la transmission. 







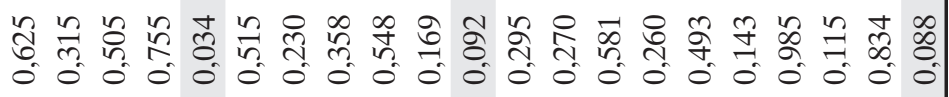

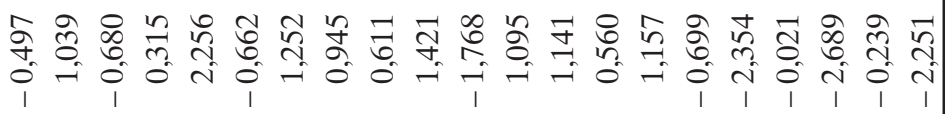

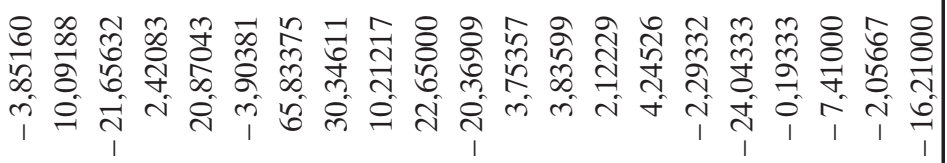



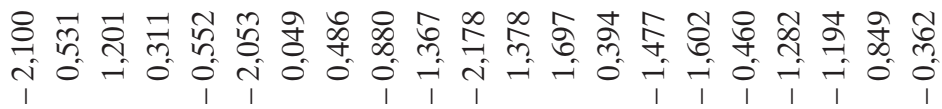

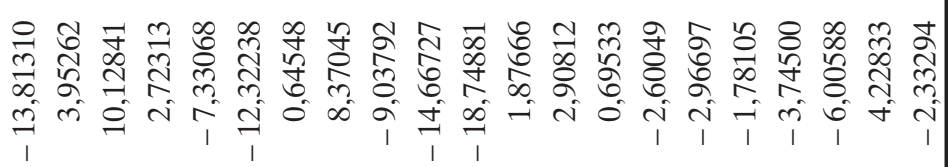

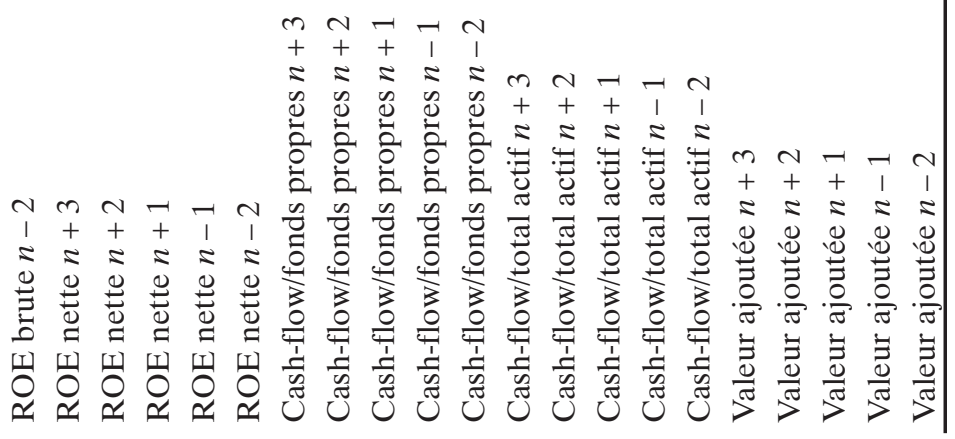

Revue internationale P.M.E., vol. 22, n² 2, 2009 


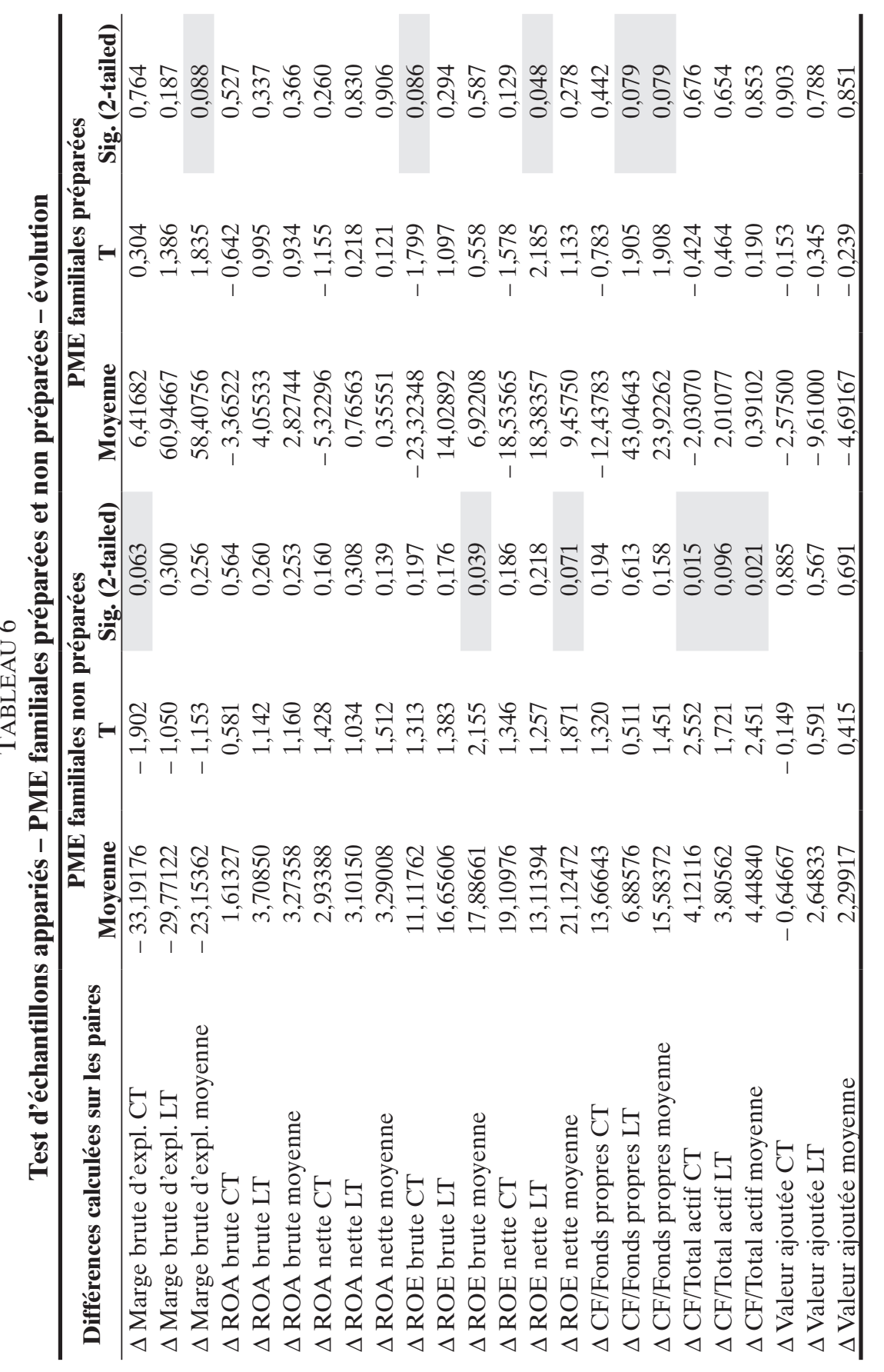






Revue internationale P.M.E., vol. 22, n² 2, 2009 
Enfin, la nature familiale des PME de notre échantillon peut laisser supposer que même s'il n'y a pas eu de réelle préparation à la transmission (calendrier, stratégie), le repreneur était déjà présent au sein de l'entreprise dans de nombreux cas (de reprises familiales) et y a acquis l'expérience et les compétences spécifiques nécessaires.

Sur ce dernier point, nous avons voulu compléter l'analyse en reconduisant les mêmes tests sur les PME non familiales transmises en les distinguant eu égard à leur degré de préparation. En effet, dans les entreprises non familiales, la probabilité de la présence préalable du repreneur au sein de l'entreprise est très faible.

Les résultats nous permettent de constater une évolution négative des ratios de performance pour les PME non familiales transmises par rapport aux PME non familiales non transmises. Nous pouvons également confirmer que l'évolution est encore plus négative lorsque la transmission n'a pas été préparée (résultat statistiquement significatif pour les évolutions en moyenne avant-après transmission du ROE, du CF/FP et du CF/TA). Pour les PME non familiales, il semble donc que la performance après la transmission varie de manière plus négative lorsque l'opération de transmission n'a pas été préparée.

\section{Conclusion}

Une large littérature conceptuelle a mis en évidence la difficulté pour les entreprises familiales à réussir leur transmission à la génération suivante et certains facteurs susceptibles d'influencer favorablement ou défavorablement le succès de la transmission.

Dans cette littérature, de nombreuses raisons sont évoquées pour expliquer ce taux d'échec si élevé. Parmi celles-ci, on peut citer l'absence ou le manque de préparation, le choix d'une technique de transmission inadaptée, les conflits familiaux et l'adhésion du personnel. La principale cause est l'absence de planification de la transmission ou une mauvaise préparation.

Cette recherche a donc été axée sur les difficultés qui se sont posées lors de la transmission et sur la préparation. L'étude empirique réalisée à partir d'un échantillon de 204 PME belges, familiales ou non, préparées ou non à la transmission, permet d'étayer ces apports conceptuels et contribue ainsi à une meilleure compréhension des mécanismes de transmission des PME familiales.

L'enquête par questionnaire portant sur 2000 PME, réalisée afin de connaître les PME familiales ainsi que les opérations de transmission, montre qu'une PME sur deux éprouve des difficultés lors de la transmission et confirme que les principaux problèmes sont bien ceux présentés dans la littérature (difficultés de trésorerie, adhésion du personnel, appareil productif obsolète ou non compétitif, endettement trop lourd, manque de préparation). 
La comparaison des performances réalisées par les PME familiales préparées à la transmission et les PME familiales non préparées ne nous a pas permis de mettre en évidence des différence sur les trois années qui suivent la transmission, au contraire des PME non familiales. L'enquête a, par contre, montré que les PME familiales éprouvent moins de difficultés lors de leur transmission que les PME non familiales (résultats limités à l'échantillon), ce qui pourrait s'expliquer par le fait que le repreneur (interne, membre de la famille) connaît l'entreprise et a déjà acquis les connaissances nécessaires à sa gestion, et en ce qui concerne ses réseaux, son personnel et sa communication.

En ce qui concerne l'évolution des performances après la transmission (observée sur trois ans), nous avons noté une performance plus faible en cas de non-préparation pour les PME devenues non familiales (résultats statistiquement significatifs).

Un certain nombre de limites doivent néanmoins être exposées. Tout d'abord, notre échantillon n'est constitué que de PME encore en activité. Dès lors, nous pouvons présumer que les PME non préparées à la transmission, qui se sont heurtées à des difficultés et qui ont cessé leurs activités durant les trois années qui ont suivi la transmission n'apparaissent pas dans nos résultats. Par ailleurs, il est fréquent de constater que, dans de nombreuses PME familiales, il n'y a pas de stratégie définie sur papier mais plutôt une préparation implicite et difficilement mesurable.

Enfin, il est très difficile d'estimer les performances des PME familiales car celles-ci ont des logiques de fonctionnement parfois très différentes de celles des entreprises managériales. Ainsi, la priorité peut être donnée à l'entreprise ou à la famille. Dans le cas d'une priorité donnée à la famille (entreprise dite de «centre de frais» ou distribution des richesses), il se peut que les fonds propres ne soient jamais alimentés. La comparaison avec une entreprise ayant opté pour la priorité entreprise et basée sur des indicateurs rapportés sur les fonds propres risque donc d'être biaisée. Dans ce papier, nous avons essayé de nuancer cette possibilité en utilisant un indicateur de rentabilité supplémentaire, à savoir le cash-flow sur le total bilantaire, en plus des indicateurs habituels de performance.

Pour conclure, certaines pistes pour des recherches futures peuvent être avancées. Il serait particulièrement intéressant de comparer, parmi les entreprises ayant fait l'objet d'une transmission, celles n'ayant rencontré aucune difficulté avec celles ayant fait faillite dans les trois à cinq années suivant la reprise, et ce, de manière à établir des profils types ou encore à mettre en évidence les points forts ou les faiblesses des transmissions. Cependant, il faudra bien définir le terme «difficultés rencontrées». Une autre piste serait de prendre les entreprises les plus performantes et de relever les principales mesures prises pour la préparation de la transmission. Mais il faut bien garder à l'esprit que chaque transmission est unique. 


\section{ANNEXE I \\ Questionnaire de l'enquête}

\section{1) Fiche signalétique de l'entreprise}

\section{Identification}

Dénomination sociale :

Adresse:

Code postal:

Ville/commune:

$\mathrm{N}^{\circ}$ TVA:

Forme juridique :

Nom du répondant:

Fonction:

Tél.:

E-mail :

Date de création de l'entreprise :

Pour l'entité concernée par vos réponses, des états financiers

sont-ils publiés auprès de la Centrale des bilans de

la Banque nationale de Belgique? (entourez)

Oui Non

\section{Activité}

1. Quelle est l'activité de votre entreprise?

\section{Dirigeants et actionnariat de l'entreprise}

2. L'entreprise est-elle dirigée par une ou plusieurs personnes ? Nombre:

3. Âge de la ou des personnes qui dirigent l'entreprise:

4. Le ou les dirigeants sont-ils actionnaires dans l'entreprise? (entourez)

5. Quelle est la structure de l'actionnariat de votre entreprise?

\begin{tabular}{|l|l|}
\hline \multicolumn{1}{|c|}{ Structure détenue } & Cochez une case \\
\hline $\begin{array}{l}\text { À } 100 \% \text { par votre famille proche } \\
\text { (vous, votre époux ou épouse, vos enfants) }\end{array}$ & \\
\hline $\begin{array}{l}\text { A } 100 \% \text { par votre famille élargie aux frères, } \\
\text { sœurs, parents, cousins, cousines }\end{array}$ & \\
\hline À plus de $90 \%$ par votre famille élargie & \\
\hline À plus de $50 \%$ par votre famille élargie & \\
\hline À plus de $50 \%$ par une ou plusieurs autres sociétés & \\
\hline Autre structure: & \\
\hline
\end{tabular}

6. La majorité des administrateurs de l'entreprise provient-elle de la famille? (entourez) 
7. Quelle est l'origine de votre entreprise?

\begin{tabular}{|l|l|}
\hline \multicolumn{1}{|c|}{ Origine } & Cochez une case \\
\hline Vous l'avez créée. & \\
\hline Vous l'avez reprise de vos parents ou beaux-parents*. & \\
\hline Vous l'avez reprise d'un membre de votre famille élargie*. & \\
\hline Vous l'avez rachetée à un ami*. & \\
\hline Vous l'avez rachetée à votre patron*. & \\
\hline Vous l'avez rachetée autrement que ci-dessus ${ }^{\star}$. & \\
\hline Autre origine: & \\
\hline
\end{tabular}

* N'oubliez pas de compléter la partie 3 juste après avoir rempli la partie 2.

\section{2) Préparation de la transmission de l'entreprise}

8. Avez-vous déjà songé à la transmission de votre entreprise? (entourez)

9. Dans combien d'années souhaitez-vous transmettre votre entreprise?

\begin{tabular}{|c|c|}
\hline Délai de transmission & Cochez une case \\
\hline Dans les 2 ans & \\
\hline Entre 2 et 5 ans & \\
\hline Entre 5 et 10 ans & \\
\hline Entre 10 et 15 ans & \\
\hline Entre 15 et 20 ans & \\
\hline Dans plus de 20 ans & \\
\hline
\end{tabular}

10. Avez-vous déjà effectué des démarches en vue de préparer la transmission de votre entreprise?

\begin{tabular}{|l|l|}
\hline \multicolumn{1}{|c|}{ Démarches effectuées } & Plusieurs choix possibles \\
\hline Aucune & \\
\hline J'en ai parlé à ma famille proche. & \\
\hline J'en ai parlé à ma famille élargie. & \\
\hline J'en ai parlé à mon comptable. & \\
\hline J'en ai parlé à mon notaire. & \\
\hline J'en ai parlé à mon banquier. & \\
\hline J'en ai parlé à mon conseiller fiscal. & \\
\hline J'en ai parlé à mon personnel. & \\
\hline J'ai consulté un organisme professionnel. & \\
\hline J'ai délégué des tâches de direction. & \\
\hline
\end{tabular}


11. Avez-vous déjà établi un plan sur la manière de gérer la transmission de votre entreprise?

\begin{tabular}{|l|l|l|}
\hline & Oui & Non \\
\hline Stratégie & & \\
\hline Calendrier & & \\
\hline
\end{tabular}

12. Connaissez-vous les facteurs de réussite dans ce domaine? (entourez)

Pouvez-vous en citer: 1)

3)

13. Des mesures concrètes ont-elles déjà été prises pour préparer la transmission?

\begin{tabular}{|l|l|l|}
\hline \multicolumn{1}{|c|}{ Mesures } & Oui & Non \\
\hline Chercher un successeur. & & \\
\hline Présenter un successeur. & & \\
\hline Former un successeur. & & \\
\hline Modifier la forme juridique de l'entreprise. & & \\
\hline Autres & & \\
\hline Pas encore de mesures concrètes. & & \\
\hline
\end{tabular}

14. Si vous connaissez déjà votre successeur idéal, avez-vous vérifié ses qualifications, son expérience? (entourez)

Oui Non

15. Est-il d'accord de reprendre l'entreprise? (entourez)

Oui Non Vous ne savez pas

16. Sinon, connaissez-vous des personnes intéressées par votre entreprise?

\begin{tabular}{|l|l|l|}
\hline & Oui & Non \\
\hline Concurrents & & \\
\hline Partenaires & & \\
\hline Travailleurs de l'entreprise & & \\
\hline Clients & & \\
\hline Autres & & \\
\hline
\end{tabular}


17. Connaissez-vous les avantages que le successeur tirerait de la reprise de votre entreprise?

\begin{tabular}{|l|l|l|}
\hline Avantages & Oui & Non \\
\hline Une clientèle & & \\
\hline Un savoir-faire & & \\
\hline Un personnel qualifié & & \\
\hline Un lieu d'implantation privilégié & & \\
\hline Un matériel performant & & \\
\hline
\end{tabular}

18. En cas de transmission familiale, le successeur exerce-t-il déjà des fonctions de direction? (entourez)

Oui Non

19. En cas de transmission familiale, le successeur a-t-il acquis de l'expérience en dehors de l'entreprise? (entourez)

Oui Non

Si oui: - de 3 ans ou + de 3 ans

20. En cas de transmission de l'entreprise à titre gratuit à un de vos enfants, voulez-vous respecter une stricte égalité envers tous vos enfants lors de la répartition de vos biens? (entourez)

21. Profitez-vous de la transmission de votre entreprise pour régler la transmission de tous vos autres biens? (entourez)

Oui Non

22. Voulez-vous travailler avec votre successeur pendant une période de transition? (entourez)

Oui Non

23. Quels sont vos besoins en information et en conseil pour les domaines suivants?

\begin{tabular}{|l|l|l|l|l|}
\hline & $\begin{array}{c}\text { Aucun } \\
\text { besoin }\end{array}$ & $\begin{array}{c}\text { Une } \\
\text { information } \\
\text { générale }\end{array}$ & $\begin{array}{c}\text { Une } \\
\text { information } \\
\text { spécifique }\end{array}$ & $\begin{array}{c}\text { Un } \\
\text { suivi } \\
\text { constant }\end{array}$ \\
\hline $\begin{array}{l}\text { Droits } \\
\text { de succession }\end{array}$ & & & & \\
\hline Droits de donation & & & & \\
\hline $\begin{array}{l}\text { Impôt des personnes } \\
\text { physiques }\end{array}$ & & & & \\
\hline Impôt des sociétés & & & & \\
\hline Droit social & & & & \\
\hline Évaluation de l'entreprise & & & & \\
\hline $\begin{array}{l}\text { Gestion des relations } \\
\text { avec la famille }\end{array}$ & & & & \\
\hline $\begin{array}{l}\text { Gestion des relations } \\
\text { avec le personnel }\end{array}$ & & & & \\
\hline $\begin{array}{l}\text { Gestion des relations avec } \\
\text { les clients, fournisseurs, } \\
\text { banquiers, etc. }\end{array}$ & & & & \\
\hline
\end{tabular}


24. Quel type de transmission allez-vous choisir?

\begin{tabular}{|c|c|c|}
\hline \multicolumn{2}{|c|}{ Type de transmission } & Cochez \\
\hline \multicolumn{3}{|c|}{ Succession de l'entreprise (voie testamentaire) } \\
\hline \multicolumn{3}{|c|}{ Donation de l'entreprise } \\
\hline \multicolumn{3}{|c|}{ Revente de l'entreprise } \\
\hline \multicolumn{2}{|c|}{ Introduction en Bourse et revente des actions } & \\
\hline \multirow{4}{*}{$\begin{array}{l}\text { Structures de } \\
\text { contrôle avec } \\
\text { don manuel } \\
\text { des titres }\end{array}$} & passage en société en commandite par actions & \\
\hline & création d'un holding & \\
\hline & création d'un bureau d'administration & \\
\hline & $\begin{array}{l}\text { division de l'entreprise en une société } \\
\text { de patrimoine et une société d'exploitation }\end{array}$ & \\
\hline \multicolumn{2}{|c|}{ Autre technique à préciser: } & \\
\hline \multicolumn{2}{|c|}{ Pas encore de technique définie } & \\
\hline \multicolumn{2}{|c|}{ Liquidation (pas de transmission) } & \\
\hline
\end{tabular}

24. Indépendamment de la transmission de votre entreprise, votre retraite est-elle assurée? (entourez)

Oui Non

25. Avez-vous une idée de ce que vous ferez après la transmission de votre entreprise? (entourez)

Oui Non

3) Réalisation de la transmission de l'entreprise

26. Votre entreprise a-t-elle déjà fait l'objet d'une transmission depuis sa création? (entourez)

Oui Non*

* Si oui, vous pouvez répondre aux questions de cette 3 e partie.

* Sinon, l'enquête se termine ici.

27. En quelle année a commencé le processus de transmission de l'entreprise?

En quelle année la transmission de l'entreprise était-elle accomplie?

28. Quel était le contexte de la transmission?

\begin{tabular}{|l|l|}
\hline & Cochez une case \\
\hline Retraite & \\
\hline Décès & \\
\hline Divorce, maladie, accident & \\
\hline Changement d'activité & \\
\hline Redistribution du capital entre actionnaires existants & \\
\hline $\begin{array}{l}\text { Raisons personnelles (retraite anticipée, } \\
\text { changement de profession, etc.) }\end{array}$ & \\
\hline Autres à préciser: & \\
\hline
\end{tabular}


29. Au cours du processus de transmission, l'entreprise a-t-elle changé de forme juridique? (entourez)

30. Quel âge avait le chef d'entreprise lors de la transmission de l'entreprise au successeur:

31. Qui est le repreneur?

\begin{tabular}{|l|l|}
\hline & Cochez une case \\
\hline Transmission à vos enfants & \\
\hline Transmission à votre famille élargie & \\
\hline Transmission aux salariés & \\
\hline Transmission à une personne extérieure à l'entreprise & \\
\hline Absence de successeur / liquidation & \\
\hline Autre repreneur: & \\
\hline
\end{tabular}

32. Profil du ou des repreneurs

Âge: $\quad$ Sexe:(entourez) Masculin Féminin

Combien de repreneurs (entourez): $\quad 1 \quad 2 \quad 3$ ou +

Le ou les repreneurs ont-ils de l'expérience

dans le domaine d'activité de l'entreprise? (entourez) Non Oui

Si oui : moins de 3 ans plus de 3 ans

Niveau d'éducation:

\begin{tabular}{|l|l|}
\hline & Cochez une case \\
\hline Universitaire & \\
\hline Supérieur de type long & \\
\hline Supérieur de type court & \\
\hline Secondaire supérieur & \\
\hline Secondaire inférieur & \\
\hline Enseignement professionnel & \\
\hline Autre: & \\
\hline
\end{tabular}

Situation antérieure du ou des repreneurs:

\begin{tabular}{|l|l|}
\hline & Cochez une case \\
\hline Salarié dans l'entreprise & \\
\hline Exerçait déjà des fonctions de direction dans l'entreprise & \\
\hline Salarié dans une autre entreprise & \\
\hline Chef d'une autre entreprise & \\
\hline Fonctionnaire & \\
\hline Chômeur & \\
\hline Autre: & \\
\hline
\end{tabular}


33. Le chef d'entreprise a-t-il travaillé avec son successeur pendant une période de transition? (entourez)

Si oui, cette période s'est étalée sur combien de temps?

34. Quel a été le type de transmission de l'entreprise?

\begin{tabular}{|c|c|c|}
\hline \multicolumn{2}{|c|}{ Type de transmission } & Cochez \\
\hline \multicolumn{2}{|c|}{ Succession de l'entreprise } & \\
\hline \multicolumn{2}{|c|}{ Donation de l'entreprise } & \\
\hline \multicolumn{2}{|c|}{ Revente de l'entreprise } & \\
\hline \multicolumn{2}{|c|}{ Introduction en Bourse et revente des actions } & \\
\hline \multirow{4}{*}{$\begin{array}{l}\text { Structures de } \\
\text { contrôle avec } \\
\text { don manuel } \\
\text { des titres }\end{array}$} & passage en société en commandite par actions & \\
\hline & création d'un holding & \\
\hline & création d'un bureau d'administration & \\
\hline & $\begin{array}{l}\text { division de l'entreprise en une société de patrimoine } \\
\text { et une société d'exploitation }\end{array}$ & \\
\hline \multicolumn{2}{|c|}{ Autres à préciser: } & \\
\hline Liquidation ( & de transmission) & \\
\hline
\end{tabular}

35. L'entreprise a-t-elle survécu à la transmission? (entourez) Oui Non

36. En cas d'échec, était-ce dans les cinq ans qui ont suivi la transmission? (entourez)

37. Quelles ont été les principales difficultés rencontrées lors de la transmission de l'entreprise?

\begin{tabular}{|l|l|}
\hline Principales difficultés rencontrées (plusieurs choix possibles) & Cochez \\
\hline Aucune & \\
\hline Difficulté de trésorerie & \\
\hline Adhésion du personnel & \\
\hline Appareil productif inadéquat, obsolète ou non compétitif & \\
\hline Endettement trop lourd & \\
\hline Manque de préparation de la transmission & \\
\hline Pas de préparation de la transmission & \\
\hline Entreprise trop influencée par la personnalité du chef d'entreprise & \\
\hline Manque d'information et de communication dans la famille & \\
\hline Pas de volonté du chef d'entreprise de passer la main & \\
\hline $\begin{array}{l}\text { Manque d'accompagnement du chef d'entreprise } \\
\text { envers son successeur }\end{array}$ & \\
\hline Manque de recours à des personnes spécialisées & \\
\hline Des héritiers ont été lésés. & \\
\hline
\end{tabular}




\begin{tabular}{|l|l|}
\hline Mode de transmission mal adapté & \\
\hline Le successeur avait une mauvaise connaissance du secteur. & \\
\hline Pas de successeur potentiel & \\
\hline $\begin{array}{l}\text { Difficultés commerciales à la suite de la perte de clients } \\
\text { ou d'un fournisseur }\end{array}$ & \\
\hline En cas de revente: valorisation trop élevée & \\
\hline En cas de revente: informations inexactes de la part du vendeur & \\
\hline Autres difficultés: & \\
\hline Autres difficultés: & \\
\hline Autres difficultés: & \\
\hline
\end{tabular}

\section{Bibliographie}

AFNOR (1988), Statistique: vocabulaire, estimation et tests statistiques, Tome 1, Paris, $435 \mathrm{p}$.

Allouche, J. et B. AmanN (2000), «L'entreprise familiale: un état de l'art», Revue Finance - Contrôle - Stratégie, vol. 3, n ${ }^{\circ}$, mars, p. 33-69.

Ambrose, D.M. (1983), «Transfer of the family-owned business », Journal of Small Business Management, janvier, p. 49-56.

AronofF, C.-E. (2001), «Understanding family-business survival statistics », Supply House Times, juillet, p. 34-35.

BARACH, J.A. et J.B. GANITSKY (1995), «Successful succession in family business », Family Business Review, vol. 8, n 2, p. 131-155.

BArbot, M.C. et K. Richomme-Huet (2004), «Proposition d'un outil de pilotage pour la transmission des PME», Colloque Métamorphoses du GREFIGE, Université Nancy II, 21 et 22 octobre, Nancy, 19 p.

BANQUE DE DÉVELOPPEMENT DES PME - BDPME (1998), «La transmission des PME-PMI, dix années d'expérience de la Banque du développement des PME», septembre, $46 \mathrm{p}$.

BECKARD, R. et G. DYER (1983), «Managing continuity in the family-owned business », Organizational Dynamics, été, p. 7-8.

BÉGIN, L. (2006), «Motivations et freins à la reprise de l'entreprise familiale en Suisse romande», Économie et Société, série K16, n 1, janvier, p. 11-36.

BIRLEY, S. (1986), «Succession in the family firm», Journal of Small Business Management, vol. 24, nº 3, juillet, p. 36-43.

BRUNELlO, T. (2002), <www.kit.brunello.net/fra/index.html>, 22 novembre 2002.

CABY, J. (1994), Motivations et efficacité des offres publiques d'achat et d'échange en France de 1970 à 1990, Thèse de doctorat, Université de Nancy II, 676 p.

CARLOCK, R.S. et J.L. WARD (2001), Strategic Planning for the Family Business. Parallel Planning to Unify the Family and Business, New York, Palgrave, 256 p. 
CHRISTENSEN, R. (1953), Management Succession in Small and Growing Enterprises, Thèse de doctorat en sciences commerciales, Boston, Harvard University, School of Business Administration, 217 p.

CHUA, J.H., P. Sharma et J.J. CHRISMAN (1999), « Succession planning and its dimension in family firms: empirical evidence», Actes $d u X V I^{e}$ Colloque annuel du Conseil canadien de la PME et de l'entrepreneuriat, Banff, Alberta, novembre, $26 \mathrm{p}$.

Colli, A. (2003), The History of Family Business 1850-2000, Cambridge, Cambridge University Press.

COMMmission EUROPÉEnNe (2004), «Rapport final du groupe d'experts sur la transmission des petites et moyennes entreprises», site <http://europa.eu.int/comm/enterprise/ entrepreneurship/support_measures/transfer_business/best_project.htm>, consulté le 25 février, 85 p.

Cooper, D.R. et P.S. Schindler (2006), Business Research Methods, 9e édition, Boston, McGraw-Hill International Edition, 744 p.

Cromie, S., B. Stephenson et D. Monteith (1995), «The management of family firms: an empirical investigation », International Small Business Journal, vol. 13, $n^{\circ} 4$, p. 11-34.

DAigne, J.F. et X. Joly (1987), Reprendre une entreprise saine, Paris, Les Éditions d'Organisation.

DAVIS, J.A. et R. TAGIURI (1989), «The influence of life stage on father-son work relationship in family companies », Family Business Review, vol. II, no 1, p. 47-74.

Diwisch, S., P. VoithofER et C. Weiss (2006), «The shadow of succession, a nonparametric matching approach », Discussion paper 13,19 p.

DONCKELS, R. (1989), À remettre entreprises familiales : la problématique de la succession, Bruxelles, Fondation Roi Baudouin, Roularta Books SA, 152 p.

DoncKels, R. et K. HOEBEKE (1991), De menselijke faktor in (familie-)KMO's, Bruxelles, KMO-studiecentrum UFSAL.

DONCKEls, R. et R. AERTs (1993), Pleins feux sur les PME, de la théorie à la pratique, Bruxelles, Fondation Roi Baudouin, Roularta Books, p. 162-164.

GAsse, Y., G. ThÉBERGE et J. NAUd (1988), «La continuité dans la PME familiale», Revue internationale PME, vol. 1, no 1, septembre, p. 43-56.

GAUTHIER, P.(1995), «Les chefs d'entreprises ne sont pas prêts à passer le flambeau», Le Magazine Québec Entreprise, octobre, p. 15-21.

HANDLER, W.-C. et K.-E. KRAM (1988), «Succession in family firms: the problem of resistance», Family Business Review, vol. 1, n4, p. 361-381.

Heldenbergh, A. (1999), Les motivations et l'efficacité des opérations stratégiques d'OPA sur le marché belge, Thèse de doctorat, Université de Mons-Hainaut, $475 \mathrm{p}$. 
HiRigoYen, G. (1988), «La transmission des PME familiales: constat et suggestions », Revue du Financier, no ${ }^{\circ}$ 5, juillet-août, p. 37-48.

INTERNATIONAL FAMILY ENTERPRISE RESEARCH ACADEMY - IFERA (2003), «Family businesses dominate», Family Business Review, vol. XVI, n 4, p. 235-239.

Jorissen, A., E. LAVEren, R. Martens et A.-M. Reheul (2002), «Differences beween family and nonfamily firms: the impact of different research samples with increasing elimination of demographic sample differences », Actes de conférence, RENT XVI, 16th workshop, 21-22 novembre, Barcelone, Espagne, Universitat Autònoma de Barcelona, 20 p.

KENYON-RouvinieZ, D. et J.L. WARD (2004), Les entreprises familiales, Paris, Presses universitaires de France, coll. «Que sais-je?», 127 p.

KITTEL, F. (1996), «La transmission d'une PME familiale», Le pouvoir dans les organisations: masques et mouvances, Groupe d'études, de recherche et d'intervention, Jean Muller (dir.), Paris, L'Harmattan, p. 173-190.

KotTer, J.P. (1982), The General Managers, New York, Free Press.

KURATKO, D.-F. (1993), «Family business succession in Korean and US firms», Journal of Small Business Management, vol. 31, n² 2, p. 132-136.

LAIDAOUI, H. (1995), «L'entreprise familiale, assurer la continuité de l'entreprise familiale», Le Magazine Québec Entreprise, octobre, p. 22.

LANGEROCK, J. (1999), «Succession dans les entreprises familiales: où le bât blesset-il ? », Bulletin de la FEB, juillet-août, n 7, p. 58-59.

LA Porta R., F. LOPEZ-DE-SilAnES et A. SHLEIFER (1999), «Corporate ownership around the world », The Journal of Finance, n $^{\circ}$ 2, p. 471-517.

LAVEREN, E. et M. BISSCHOPS (2004), «De opvolgingsproblematiek in Vlaamse bedrijven: resultaten van een empirisch onderzoek», Onderzoeksrapport UAMS, juillet, $30 \mathrm{p}$.

LEACH, P. et T. Bogod (1999), Guide to the Family Business, Londres, Kogan Page, p. 161.

LEKER, J. et S. SALOMO (2000), «CEO turnover and corporate performance», Scandinavian Journal of Management, vol. 16, nº 3, septembre, p. 287-303.

MeIER, O. (2002), «Problèmes de succession dans les PME familiales: freins et résistance culturelle », Gestion 2000, juillet-août, p. 109-126.

Morck, R. et B. Yeung (2003), «Agency problems in large family business groups », Entrepreneurship Theory and Practice, vol. 27, n 4, p. 367-382.

Mouline, J.P.(2000), «Dynamique de la succession managériale dans la PME familiale non-cotée », Revue Finance - Contrôle - Stratégie, vol. 3, nº 1, mars, p. 197-222.

NoËL, K. (1998), «80\% des chefs de PME n'ont pas de plan de succession», Les Affaires, samedi 24 octobre, p. 40.

Ooghe, H. et C. VAN Wymeersch (1990), Traité d'analyse financière, Namur, Belgique, Presses universitaires de Namur, 4e édition, tome 1, 423 p., tome 2, $110 \mathrm{p}$. 
OSEO BDPME (2005), La transmission des petites et moyennes entreprises, l'expérience d'OSEO BDPME, $68 \mathrm{p}$.

PAILOT, P. (1998), «Propositions théoriques et épistémologiques pour une méthodologie d'analyse des freins psychologiques des dirigeants de PME lors des transmissions d'entreprise», CIFPME, IAE de Metz, octobre, 21 p.

PARRINO, R. (1997), «CEO turnover and outside succession a cross-sectional analysis », Journal of Financial Economics, vol. 46, n² 2, novembre, p. 165-197.

PEAY, T.R. et J.W. GIBB DYER (1989), «Power orientations of entrepreneurs and succession planning », Journal of Small Business Management, janvier, p. 47-52.

Sharma, P., J. CHRistman et J. CHUOO (1998), «Dimensions of succession planning in family firms : an empirical assessment », Communication, ASAC.

Sonnenfeld, J. (1988), The Hero's Farewell, New York, Oxford Press.

THIÉTART, R.-A. (1999), Méthodes de recherche en management, Paris, Dunod, 535 p.

VAN CAILliE, D. (1999), «La PME et l'entreprise familiale», Séminaire sur les PME organisé par l'Institut des réviseurs d'entreprise, 62 p.

VAn Gils, A., W. Voordeckers et J. VAn Den Heuvel (2004), «Environmental uncertainty and strategic behavior in Belgian family firms », European Management Journal, vol. 22, n ${ }^{\circ}$, p. 588-595.

VATTEVILlE, E. (1994), «Le risque successoral», Revue française de gestion, marsavril-mai, no 98 , p. 18-27.

WARD, J. (1988), «The special role of strategic planning for family business », Family Business Review, vol. 1, n² 2, p. 105-117.

WARD, J. (1997), «Keeping the family business healthy. How to plan for continuing growth, profitability, and family leadership», Business Owner Resources, p. 2.

WestheAD, P. et M. COWLING (1998), «Family firm researchs: the need for a methodological rethink», Entrepreneurship, Theory and Practice, p. 31-56.

WtTerwulghe, R., F. Janssen, S. Mertens et F. Olivier (1994), Le financement de croissance des entreprises moyennes belges par le recours au capital à risque, Louvain-La-Neuve, IAG. 